\title{
HST observations rule out the association between Cir X-1 and SNR G321.9-0.3*
}

\author{
R. P. Mignani ${ }^{1}$, A. De Luca ${ }^{2,3}$, P. A. Caraveo ${ }^{2}$, and I. F. Mirabel ${ }^{4,5}$ \\ 1 ESO, Karl Schwarzschild Str. 2, 85748 Garching bei München, Germany \\ 2 Istituto di Fisica Cosmica del CNR "G. Occhialini", Via Bassini 15, 20133 Milan, Italy \\ 3 Universitá di Milano Bicocca, Dipartimento di Fisica, Piazza della Scienza 3, 20126 Milano, Italy \\ 4 Service d'Astrophysique / CEA, CE-Saclay, 91191 Gif-sur-Yvette, France \\ 5 Instituto de Astronomía y Física del Espacio/CONICET. cc5, 1428 Bs As, Argentina
}

Received 21 December 2001 / Accepted 5 February 2002

\begin{abstract}
Cir X-1 is one of the most intriguing galactic X-ray sources. It is a $\sim 16.6$ days variable $\mathrm{X} /$ radio source, a type-I X-ray burster and a QPO emitter. In spite of an uncertain optical counterpart classification, all these properties identify the source as an LMXB. The morphology of the surrounding radio nebula has suggested an association with the nearby ( $\sim 25$ arcmin) SNR G321.9-0.3, implying that Cir X-1 is a runaway binary originated from the supernova explosion $\approx 10^{5}$ years ago. To investigate this hypothesis, we carried out a proper motion measurement of the Cir X-1 $m \sim 19$ optical counterpart using a set of HST/WFC and WFPC2 observations

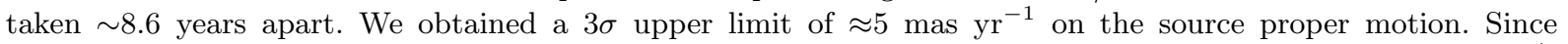

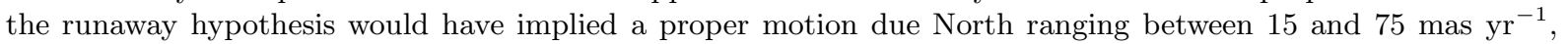
depending on the actual age of the SNR, our result definitively rules out the association between Cir X-1 and SNR G321.9-0.3.
\end{abstract}

Key words. X-ray: binaries - ISM: individual objects: SNR G321.9-0.3 - stars: individual: Cir X-1

\section{Introduction}

Cir X-1 is a strong and persistently bright galactic X-ray source with a flux modulated over a 16.6 days cycle (Kaluzienski et al. 1976; Bignami et al. 1977), which is commonly believed to coincide with the orbital period of a binary system in a highly eccentric orbit (Murdin et al. 1980). Type-I X-ray bursts have been discovered by EXOSAT (Tennant et al. 1986a,b), as well as high/low frequency QPO (Tennant 1987, 1988) whose properties suggested that Cir X-1 might represent an ideal link between the classes of the canonical $Z$ and atoll-type sources (Oosterbroek et al. 1995). In addition, long-term variations in the X-ray flux have been observed, probably due to a partial obscuration of the central source by the precession of a warped accretion disk (Oosterbroek et al. 1995). Recently, X-ray spectral evolution of Cir X-1 along the orbital cycle has been reported by Iaria et al. (2001).

While the general X-ray properties of Cir X-1 (e.g. type-I bursts, QPOs) point towards a LMXB, its nature

Send offprint requests to: R. P. Mignani, e-mail: rmignani@eso.org

* Based on observations with the NASA/ESA Hubble Space Telescope, obtained at the Space Telescope Science Institute, which is operated by AURA, Inc. under contract No NAS 5-26555. is still unclear. Although an optical counterpart has been identified (Moneti 1992; Mignani et al. 1997), no reliable spectral classification has been possible so far. The spectrum is dominated by a broad, asymmetric, $\mathrm{H} \alpha$ line (Duncan et al. 1993; Mignani et al. 1997) with no prominent stellar emission/absorption features (Johnston et al. 1999).

The radio counterpart of Cir X-1 was identified soon after the X-ray detection (Clark et al. 1975) and found to be variable with the same 16 day period (Haynes et al. 1978). The radio picture features a point source which is embedded in a $\simeq 5 \times 10 \operatorname{arcmin}^{2}$ nebula and is apparently the origin of elongated structures, extending on both arcmin (Stewart et al. 1993) and arcsec scales (Fender et al. 1998). Such a morphology has been interpreted as a jetpowered synchrotron nebula, making Cir X-1 the second galactic X-ray binary, besides SS433, which shows both radio jets and a radio synchrotron nebula.

The shape of the radio nebula, protruding southward and pointing to the center of the nearby $(\sim 25 \operatorname{arcmin})$ radio SNR G321.9-0.3, is reminiscent of a residual wake left behind as the point source moves away from the SNR (see Fig. 1, reproduced from Fig. 1 of Stewart et al. 1993). This suggested a tantalizing scenario where Cir X-1 is a runaway binary system, with the neutron star member ejected by the supernova explosion responsible for 


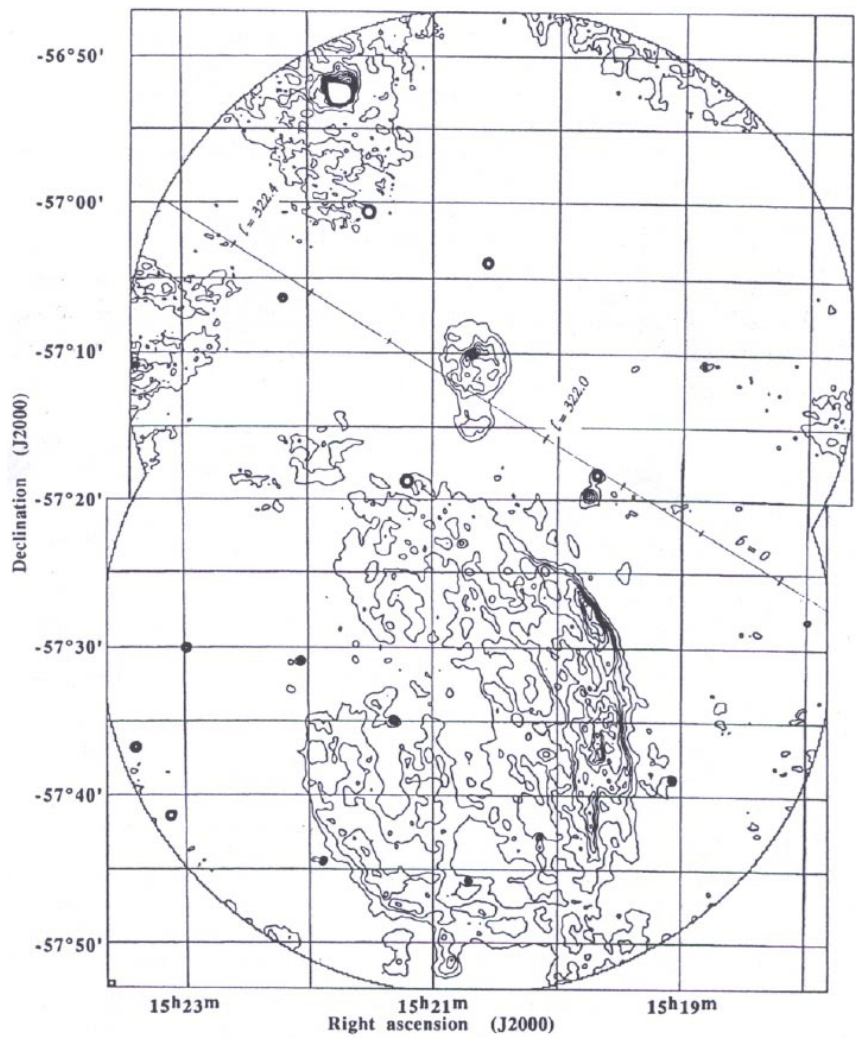

Fig. 1. ATCA radio map of the Cir X-1 region, adapted from Fig. 1 of Stewart et al. (1993). Cir X-1 is at the center of the compact radio nebula recognizable near the center of the field, with SNR G321.9-0.3 due south of it.

SNR G321.9-0.3 (Stewart et al. 1993) between $2 \times 10^{4}$ and $10^{5}$ yrs ago (Clark et al. 1975). Such a scenario is consistent, within the uncertainties, with the estimated distances of both Cir X-1 and of the SNR. From H I absorption measurements (Goss \& Mebold 1977), the distance to $\mathrm{Cir} \mathrm{X}-1$ is anywhere between 6.7 and $8 \mathrm{kpc}$, while the distance to the SNR ranges from $5.5 \mathrm{kpc}$ (Case \& Battacharya 1998) to $6.5 \mathrm{kpc}$ (Stewart et al. 1993). If the above scenario is correct, for a SNR age $T$ (yrs), Cir X-1 should move Northward in the plane of the sky with a

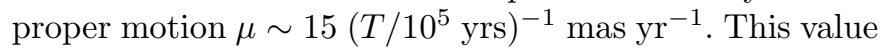
would imply a tranverse velocity with respect to the center of the SNR of $v \sim 5 \mu d_{\mathrm{kpc}} \mathrm{km} \mathrm{s}^{-1}$, where $\mu$ is in units of mas $\mathrm{yr}^{-1}$ and $d_{\mathrm{kpc}}$ is the SNR distance in units of $\mathrm{kpc}$.

From the combination of all the possible values of both the SNR age and distance, we would thus expect Cir X-1 to move Northward with a proper motion ranging between

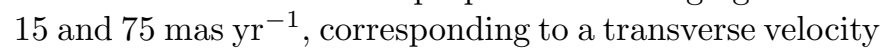
between 400 and $2400 \mathrm{~km} \mathrm{~s}^{-1}$.

Thus, the Cir X-1 angular displacement is a simple, yet powerful, tool to prove or disprove its association with SNR G321.9-0.3. Measuring a Northward angular

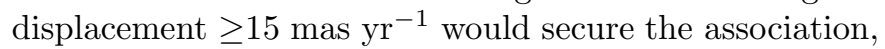
while the lack of such a displacement, or a displacement in a different direction, would rule it out. In principle, both the optical and radio counterpart could be used to gauge the precise position of the source. However, the dramatic fading of the radio point source (Stewart et al. 1991) hampers a measurement in the radio band and leaves the burden of the proof to astrometry of the optical counterpart.

The sharpness of view of HST makes it the ideal instrument to settle such a proper motion issue. Thus, we have taken advantage of an HST/WFC archival image of the field as well as of recently acquired WFPC2 ones to carry out the proper motion measurement. The observations and data reduction are described in Sect. 2 while the results are presented and discussed in Sects. 3 and 4.

\section{Observations.}

\subsection{The data sets}

The first image of the Cir X-1 field was obtained with the pre-repair HST on October $20^{\text {th }} 1992$ as a target acquisition image for spectroscopy observations with the Faint Object Spectrograph (Mignani et al. 1997). The observation was performed with the Wide Field Camera (WFC) $\left(00^{\prime \prime} 1 /\right.$ pixel $)$ through the wide band filter $785 \mathrm{LP}$ " $I$-band filter" $(\lambda=9703 \AA ; \Delta \lambda=1669.5 \AA)$, where the source is brighter $\left(m_{785 L P} \simeq 18\right)$. The exposure time was $300 \mathrm{~s}$. A new observation of Cir X-1 was performed on May $19^{\text {th }} 2001$ with the Wide Field and Planetary Camera 2 (WFPC2), as a snapshot programme. In order to achieve the highest positional accuracy, the target was positioned at the center of the Planetary Camera (PC) chip $\left(00^{\prime \prime} 045 /\right.$ pixel $)$. For consistency with the first observation, the $785 L P$ filter was used. Three observations of $300 \mathrm{~s}$ each were obtained to allow for a better cosmic ray filtering. After the standard HST pipeline reduction (debiassing, dark removal, flatfielding) the 2001 images have been combined with a median filter and stacked (Fig. 2). Residual cosmic ray hits were filtered out using specific IRAF/STSDAS routines.

\subsection{The data analysis}

The cleaned images are the starting point for our astrometric analysis. Following a robust method successfully applied in several previous astrometric works (see e.g. Caraveo et al. 1996; Caraveo \& Mignani 1999; De Luca et al. 2000, 2001; Mignani et al. 2000, 2001; Caraveo et al. 2001), this relies on an accurate image superposition which is obtained by optimizing a linear coordinate transformation between two reference grids determined by a set of common reference objects.

In order to obtain an accurate position determination, the reference objects have to be carefully selected i.e. they must be (i) not too close to the CCD edges, (ii) not extended, (iii) not saturated but not too faint. In order to avoid problems with the inter-chip astrometry (see Caraveo \& Mignani 1999), the selecton of the reference objects was limited to the chip where the target star was imaged, i.e. WFC \#2 in the 1992 observation and PC in the 2001 one. In the rather crowded field of 


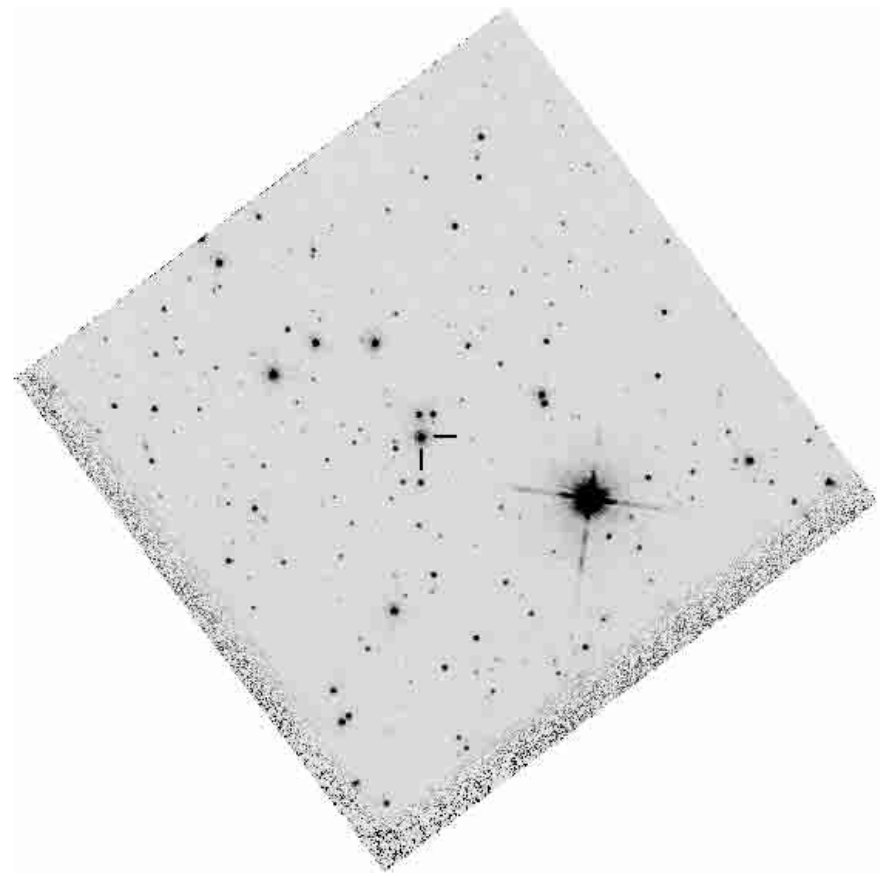

Fig. 2. WFPC2 image of the Cir X-1 field taken in 2001 with the WFPC2 through the F785LP filter. Only the PC field is shown. North is up, East is left. The two ticks mark the position of the companion star of the X-ray source.

Cir X-1, 46 good reference sources, matching the above constraints, could be found. Their positions were computed with a 2-D Gaussian fitting of their intensity profiles using centering boxes optimized in order to obtain stable values for both the centroids coordinates and their uncertainties (see De Luca et al. 2001 for a detailed discussion of both statistical and systematic errors involved in the procedure). The reference objects were positioned with an accuracy between 0.05 and 0.08 pixel (i.e. between 5 and 8 mas) in the WFC image and between 0.05 and 0.1 pixel (i.e. between 2.3 and 4.5 mas) in the PC one, depending on the object's $\mathrm{S} / \mathrm{N}$. The position of the target star was computed in the same way, with an accuracy of about 0.07 pixel ( 7 mas) and of about 0.06 pixel $(\sim 3$ mas) in the WFC and in the PC image, respectively. We note that the uncertainties on the centroids in both the WFC and the PC images are somewhat higher than the ones measured in other fields (see e.g. De Luca et al. 2000). This can be ascribed, at least in part, to the peculiar PSF in the long-pass F785LP filter, characterized by broad and relatively bright wings. This is the same effect observed in filter F1042M (it is known as the "F1042M PSF anomaly") and it is due to the CCD detector becoming transparent at longer wavelengths, so that light is reflected and scattered by the back of the CCD producing a defocused halo (see WFPC2 Instrument Handbook for further details). In addition, for the 2001 snapshot observations the analysis of the spacecraft attitude showed a jitter of the telescope about 2 times larger than average $(\approx 5$ mas rms compared to 3 mas rms) and distributed along a preferential direction. Moreover, we note that the 2001 observations were performed during the day/night transition, which occurred during the first exposure. Thermal effects, such as "breathing", could thus be present, determining a slight defocussing of the camera. All these effects clearly influence the PSF, thus affecting the centering accuracy.

The derived sets of coordinates were then corrected for known systematics, like the " $34^{\text {th }}$ row defect" of the PC (Anderson \& King 1999), and for the effects of the distortions of the WFC and the PC. While for the WFC only the geometric transformation of Gilmozzi et al. (1992) is available, for the WFPC2 we have used the most recent one provided by Casertano \& Wiggs (2000), which accounts for the evolution of the distortion map of the WFPC2 due to the drift of the instrument in the Optical Telescope Assembly focal plane.

The superposition of the frames was performed using the PC image as reference. First, the PC image was aligned in Right Ascension and Declination, according to the telescope roll angle. Then, the WFC reference grid was registered on the $\mathrm{PC}$ one by fitting a linear coordinate transformation, accounting for 2 independent scale factors, 2 translation factors and a rotation angle. Following Caraveo et al. (2001), we checked for systematics due, e.g., to unknown displacements of the reference objects by repeating the frame superposition 46 times excluding, in turn, one of our reference stars. Four objects yielding relatively high deviations (between 0.5 and $1 \mathrm{PC}$ pixel per coordinate) were thus identified and rejected. Different centering algorithms (e.g. separate Gaussian fitting on the $X$ and $Y$ intensity distributions) and coordinate transformation routines were also tested. In all cases we obtained statistically indistinguishable results, showing that our procedure is correct and free of systematics.

The final frame registration yielded a rms values on the residuals of the reference objects' coordinates of $0.33 \mathrm{PC}$ pixel (15 mas) in Right Ascension and 0.25 PC pixel (11.4 mas) in Declination. Such rms values are a factor of 4 higher than those obtained by De Luca et al. (2000) for a WFC-to-PC superposition in the Vela pulsar field. While the higher uncertainties in the centroids determination addressed above play a role, they can not account entirely for the larger residuals obtained in the image registration. This effect might be related to the likely dependance on the filter wavelength of the transformation coefficients used in the geometric correction. Unfortunately, since such dependance has been characterized for the WFPC2 (Trauger et al. 1995) but not for the WFC, the accuracy of the image registration might be affected by a non-optimized geometric correction.

\section{Results}

We have plotted in Fig. 3 the relative positions of our target together with the residuals on the reference stars positions. Since the difference between the positions of Cir X-1 is comparable with the residuals measured for the 42 reference stars, we conclude that the Cir X-1 


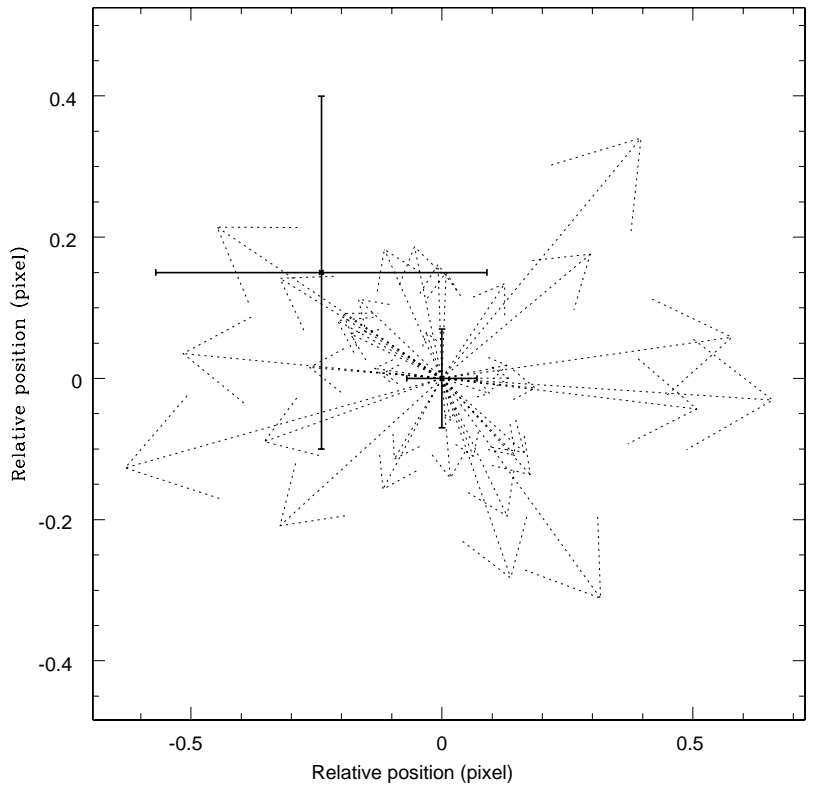

Fig. 3. Relative positions (crosses) of the Cir X-1 companion star measured at the epoch of our two observation. The error bars for the 2001 position (defining the 0,0 in the plot) account only for the centering uncertainty, while for the 1992 position they account also for the frame registration uncertainty. We overplotted as dashed arrows the residuals on the reference stars coordinates after the image superposition. Their rms value represents the frame registration uncertainty (see text). The displacement of Cir X-1 is comparable with the residuals of the reference stars.

counterpart does not show any significant displacement. The position offsets amount to $0.24 \pm 0.33 \mathrm{PC}$ pixel in RA and $-0.15 \pm 0.25 \mathrm{PC}$ pixel in Dec. By applying the nominal plate scale tranformation (PC pixel size 0 ". 0455 ), these values translate into an angular displacement in the plane of the sky of $-11 \pm 15$ mas in RA and $-7 \pm 11$ mas in Dec (neglecting the correction for the Sun's proper motion). Although such displacements are statistically null, it is worth comparing the derived proper motion with the predicted values. Over the time interval of 8.6 years spanned by our HST observations, we obtain a total yearly dis-

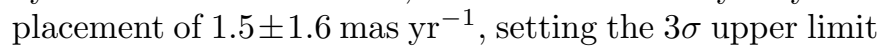
on the proper motion to $\approx 5$ mas $\mathrm{yr}^{-1}$. Such a value rules out even the minimum value of $\approx 15$ mas $\mathrm{yr}^{-1}$ required by the proposed association with G321.9-0.3 (see Sect. 1). Moreover, we note that also the derived constraints on the possible proper motion directions (Fig. 3) are incompatible with the relative positions of Cir X-1 and the SNR center, which are roughly aligned North-South (see Fig. 1). Thus, we conclude that, contrary to earlier speculations, Cir X-1 and SNR G321.9-0.3 are not associated.

\section{Discussion}

Both the lack of a measurable proper motion for Cir X-1 and its resulting non-association with SNR G321.9-0.3 have interesting astrophysical implications. Firstly, it is important to note that our result frees the neutron star age from the tight constraints imposed by the proposed SNR association. Indeed, an age greater than 100000 years would be more easily compatible with the estimated time scales of the accretion-driven decay of the neutron star magnetic field (see, e.g., Konar \& Bhattacharya 1997, 1999 and reference therein) down to the values expected for the occurance of type I X-ray bursts (Lewin \& Joss 1983).

As far as the dynamics of the binary system are concerned, the null proper motion measurement sets a $3 \sigma$ upper limit of $24 d_{\mathrm{kpc}} \mathrm{km} \mathrm{s}^{-1}$ on its relative tranverse velocity, where $d_{\mathrm{kpc}}$ is the source distance in kpc. Such a value would imply that Cir X-1 is not moving with an anomalous velocity larger than, e.g., $400 \mathrm{~km} \mathrm{~s}^{-1}$ unless its spatial velocity is essentially radial. A possible indication for an high radial velocity $\left(\approx 430 \mathrm{~km} \mathrm{~s}^{-1}\right)$ was indeed found in the spectroscopic observations of the Cir X-1 optical counterpart (Johnston et al. 1999), although it could not be unambiguously confirmed by the more recent work of Johnston et al. (2001). Together with our constraint on the tranverse velocity, an accurate spectroscopic measurement of the radial velocity would be critical to determine the dynamics of the binary system and to compute the kick velocity impressed by the SN explosion (Tauris et al. 1999). Moreover, the above constraints on the binary system dynamics may require a different scenario for the source interactions with the surrounding environment. In particular, the lack of a proper motion in the expected direction prompts for an alternative explanation for the morphology of the arc-shaped radio features observed in the synchrotron nebula, so far interpreted as jets trailing behind by a fast-moving source.

Last, but not least, our result calls, once again, for more caution in associating compact objects with SNRs purely on the basis of their proximity in the plane of the sky and on morphological arguments.

Acknowledgements. We thank the referee, H. Johnston, for her useful comments. We are also grateful to G. F. Bignami for his critical reading of the manuscript.

\section{References}

Anderson, J., \& King, I. R. 1999, PASP, 111, 1095

Bignami, G. F., della Ventura, A., Maccagni, D., \& Stigliz, R. 1977, A\&A, 57, 309

Caraveo, P. A., Bignami, G. F, Mignani, R., \& Taff, L. 1996, ApJ, L461, L91

Caraveo, P. A., \& Mignani, R. P. 1999, A\&A, 344, 367

Caraveo, P. A., DeLuca, A., Mignani, R. P., \& Bignami, G. F. 2001, ApJ, 561, 930

Case, G. L., \& Bhattacharya, D. 1998, ApJ, 504, 761

Casertano, S., \& Wiggs, M. S. 2000, WFPC2 Instrument Handbook v5.0, 2000, Ed. STScI

Clark, D. H., Caswell, J. L., \& Green, A. J. 1975, Aust. J. Phys. Astrophys. Suppl., 37, 1

Clark, D. H., Parkinson, J. H., \& Caswell, J. L., 1975, Nature, 254, 674

De Luca, A., Mignani, R. P., \& Caraveo, P. A. 2000, A\&A, 354, 1011 
De Luca, A., Mignani, R. P., \& Caraveo, P. A. 2001, Proc. of the Symp: A Decade of HST Science, Baltimore, April 11-14 2000, ed. M. Livio, K. Noll, \& M. Stiavelli, p. 32 [astro-ph/0009034]

Duncan, A. R., Stewart, R. T., \& Haynes, R. F. 1993, MNRAS, 265, 157

Fender, R. P., et al. 1998, ApJ, 506, L21

Goss, W. M., \& Mebold, U. 1977, MNRAS, 181, 255

Gilmozzi, R., Bwald, S., \& Kinney, B. 1992, WFPC2 Instrument Science Report 95-02

Haynes, R. F., et al. 1978, MNRAS, 185, 661

Johnston, H. M., Fender, R., \& Wu, K. 1999, MNRAS, 308, 415

Iaria, R., Di Salvo, T., Burderi, L., \& Robba, N. R. 2001, ApJ, 561,321

Johnston, H. M., Wu, K., Fender, R., \& Cullen, J. G. 2001, MNRAS, 328, 1193

Kaluzienski, L. J., Holt, S. S., Boldt, E. A., \& Serlemitsos, P. J. 1976, ApJ, 208, L71

Lewin, W. H. G., \& Joss, P. C. 1983, in Accretion Driven Stellar X-ray Sources, ed. W. H. G. Lewin, \& E. P. J. van den Heuvel (Cambridge University Press, Cambridge)

Mignani, R. Caraveo, P. A., \& Bignami, G. F. 1997, A\&A, 323, 797

Mignani, R. P., De Luca, A., \& Caraveo, P. A. 2000, ApJ, 543, 318
Mignani, R. P., Caraveo, P. A., \& De Luca, A. 2001, Proc. of the Symp: A Decade of HST Science, Baltimore, April 11 - 14 2000, ed. M. Livio, K. Noll, \& M. Stiavelli, p. 65 [astro-ph/0009033]

Moneti, A. 1992, A\&A, 260, L7

Murdin, P., et al. 1980, A\&A, 87, 292

Osterbroek, T., Van der Klis, M., Kuulkers, E., Van Paradijs, J., \& Lewin, W. H. G. 1995, A\&A, 297, 141

Stewart, R. T., et al., 1991, MNRAS, 253, 212

Stewart, R. T., Caswell, J. L., Haynes, R. F., \& Nelson, G. J. 1993, MNRAS, 261, 593

Tauris, T. M, Fender, R. P., van den Heuvel, E. P. J., Johnston, H. M., \& Wu, K. 1999, MNRAS, 310, 1165

Tennant, A. F. 1987, MNRAS, 226, 971

Tennant, A. F. 1988, MNRAS, 230, 403

Tennant, A. F., Fabian, A. C., \& Shafer, R. A., 1986a, MNRAS, 219,871

Tennant, A. F., Fabian, A. C., \& Shafer, R. A. 1986b, MNRAS, 221,27

Trauger, J. T., Vaughan, A. H., Evans, R. W., \& Moody, D. C. 1995, in Proc. of the Workshop: Calibrating Hubble Space Telescope. Post Servicing Mission, ed. A. Koratkar, Space Telescope Science Institute Pubs., 379 